\title{
Propiedades psicométricas de la versión española del Marihuana Motives Measure en población adolescente consumidora
}

\section{Spanish version validation of the Marihuana Motives Measure in a drug-consuming adolescent sample}

\author{
J.L. Matali*, J. Simons**, M. Pardo*, M. Lleras*, A. Pérez*, O. Andión*** \\ *Servicio de Psiquiatría y Psicología. Hospital Sant Joan de Déu de Barcelona. Grupo de investigación en trastornos mentales \\ en la infancia y la adolescencia. Institut de Recerca Sant Joan de Déu (Spain). \\ **Department of psychology. University of South Dakota (EEUU). \\ ***Departamento de Psiquiatría. Hospital Universitari Vall d'Hebron, Barcelona, CIBERSAM.
}

\section{Resumen}

Introducción: El cannabis es la sustancia ilegal que más consumen los adolescentes españoles. Entender los motivos de consumo es un factor importante para la intervención. Actualmente no existe en España un instrumento para su evaluación. El objetivo del presente trabajo es estudiar las propiedades psicométricas de la versión española del cuestionario Marihuana Motives Measure (MMM) en una población de adolescentes consumidores.

Material y Métodos: Se llevó a cabo una traducción y retrotraducción del MMM. Un total de 228 adolescentes consumidores de cánnabis fueron evaluados. Se realizó un análisis factorial y se estudió la fiabilidad de la puntuación total y de cada una de las escalas del cuestionario a partir del Alfa de Cronbach. El estudio de la evidencia de validez del MMM se realizó mediante el examen de las correlaciones entre el uso actual de cánnabis, los efectos subjetivos del consumo través del cuestionario ARCI (Addiction Research Center Inventory) y la personalidad, mediante el cuestionario MACI (Millon Adolescent Clinical Inventory).

Resultados: Se observó una alta fiabilidad de la puntuación total del MMM (Alfa de Cronbach=0,86) y entre alta y moderada para cada uno de los cinco factores obtenidos al realizar el análisis factorial del MMM, Social =0,82; Enhancement $=0,72 ;$ Coping $=0,83 ;$ Expansion $=0,74$; Conformity $=0,64$. Además, se observaron correlaciones significativas tanto entre motivos de consumo de cannabis y efectos subjetivos, así como entre motivos de consumo y personalidad.

Conclusiones: La versión española del MMM muestra una estructura factorial similar a la obtenida por el autor original y sus medidas resultan fiables y válidas para el estudio de los motivos de consumo de cánnabis en población adolescente consumidora.

Palabras clave: Adolescentes; adicción; cannabis; motivos; Marihuana Motives Measure.

\section{Abstract}

Introduction: Cannabis is the illicit drug mostly widely consumed by adolescents in Spain. The understanding of consumption motives is an important factor for intervention. In Spain, there are no available instruments for their evaluation, hence, the goal of this paper is to study the psychometric properties of the Marihuana Motives Measure (MMM) in a sample of adolescent consumers.

Material and Method: Firstly, translation and back-translation was performed. A total of 228 adolescent consumers of cannabis were evaluated. Factorial analysis was conducted, and the reliability of the total scores and of each scale of the questionnaire was studied through Cronbach's alpha. Test-retest reliability was analyzed through interclass correlations. Validity evidence of the MMM was examined through correlations between current cannabis use, subjective consumption effects measured with the Addiction Research Center Inventory (ARCI), and personality measured with the Millon Adolescent Clinical Inventory (MACI).

Results: High reliability was observed in total score of the MMM (Cronbach $\alpha=.86$ ), and high and moderate reliability for each of the five factors obtained in the factorial analysis of the MMM, Social $=.82$, Enhancement $=.72$, Coping $=.83$, Expansion $=.74$, and Conformity $=.64$. Significant correlations were also observed between cannabis consumption motives and subjective effects, and between consumption motives and personality.

Conclusion: The Spanish version of the MMM shows a similar factorial structure as the one obtained by the original author, and its measures are reliable and valid for the study of cannabis consumption motives in adolescent consumer population.

Keywords: Adolescents; addiction; cannabis; motives; Marihuana Motives Measure. 


\section{Introducción}

$\mathrm{E}$ 1 cannabis es la droga ilegal más consumida por los adolescentes españoles (ESTUDES, 2014). Además, en la actualidad, el consumo de cannabis es una de las causas que genera en España una mayor demanda de tratamiento psiquiátrico en este grupo de edad (ESTUDES, 2014) y la más presente en los pacientes que acuden a urgencias psiquiátricas infanto-juveniles (Arias Constantí et al., 2010). Esta situación es especialmente preocupante si tenemos en cuenta la constatada evidencia sobre las múltiples consecuencias negativas asociadas al consumo (Degenhardt et al., 2010; Fox, Towe, Stephens, Walker y Roffman, 2011) siendo la repercusión escolar una de las principales (Horwood et al., 2010).

Posiblemente, como consecuencia de este incremento de adolescentes consumidores de cannabis y de las graves repercusiones que su consumo tiene en ellos, en los últimos años se ha observado un aumento de los estudios sobre la efectividad de los tratamientos a aplicar (Fox, Towe, Stephens, Walker y Roffman, 2011). Entre las modalidades estudiadas cabe destacar las intervenciones basadas en las estrategias motivacionales que han demostrado ser efectivas en el abordaje de los adolescentes consumidores (Fox, Towe, Stephens, Walker y Roffman, 2011). Los modelos motivacionales del uso de sustancias sugieren que la conducta está generada por diferentes razones y considera los motivos de consumo de vital importancia a la hora de entender el contexto y las circunstancias del comportamiento adictivo, como cuándo o dónde consumir o en qué frecuencia o cantidad (Cooper, 1994).

Tomando como punto de referencia el modelo motivacional, Simons, Correia, Carey y Borsari (1998), desarrollaron el Marihuana Motives Measure (MMM) con la intención de poder cuantificar los motivos de consumo del cannabis. El estudio de las propiedades psicométricas del MMM indicó que podía ser agrupado en 5 subescalas o factores que presentan una consistencia interna entre 0,85 y 0,93. Las escalas originales fueron denominadas: 1) Enhancement "Consumo para mejorar los sentimientos positivos", 2) Social "Consumo para mejorar el refuerzo y la cohesión social", 3) Coping "Consumo para afrontar las emociones negativas", 4) Conformity "Consumo para evitar el rechazo social" y 5) Expansion "Consumo para ampliar la conciencia”. Las diferentes escalas o motivos incluidos en el MMM han demostrado ser predictores de problemas relacionados con el consumo de cánnabis (Simons, Correia y Carey, 2000). Concretamente, los estudios muestran la importancia de los motivos de consumo Social y Conformity como predictores de las consecuencias derivadas del consumo (Simons, Gaher, Correia, Hansen y Christopher, 2005), así como una correlación positiva entre la cantidad de motivos de consumo reconocidos y una mayor gravedad (Zvolensky et al. 2007). Además, las propiedades psicométricas del
MMM han sido estudiadas en muestras no anglosajonas (Chabrol, Ducongé, Casas, Roura y Carey, 2005), confirmando la estructura de 5 factores y una alta validez interna del cuestionario.

Los motivos de consumo han sido estudiados principalmente siguiendo dos líneas de investigación. La primera línea, centrada en el consumo y la percepción de reducción del malestar, muestra una asociación consistente entre los efectos subjetivos descritos por los consumidores de cánnabis y los motivos que dan para utilizarlo, por ejemplo la motivación de consumir para afrontar una situación que le preocupa y el efecto reportado de relajación (Dekker, Linszen y De Haan, 2009; Scherrer et al. 2009; Zeiger et al., 2012). La segunda línea de estudio tiene como objetivo analizar la relación entre los motivos de consumo y la personalidad (Tragesser, Trull, Sher y Park, 2008; Littlefield, Sher y Wood 2010a; Littlefield, Sher y Wood 2010b). Estos estudios muestran que los motivos de consumo son un claro mediador entre la personalidad y el consumo (Adams, Kaiser, Lynam, Charnigo y Milich, 2012). Concretamente, los factores de personalidad relacionados con la afectividad negativa están mayoritariamente asociados con el consumo de cannabis y otras sustancias por motivos de Coping (Mezquita et al., 2011). Contrariamente, los factores de personalidad relacionados con la extraversión, la búsqueda de sensaciones y la impulsividad se asocian sobre todo con el consumo de cannabis y otras sustancias por motivos de Enhancement (Mezquita et al., 2011).

La evidencia mencionada en los párrafos anteriores enfatiza la necesidad de identificar y mejorar la comprensión de los motivos de consumo en los adolescentes. Además, remarca la importancia de tenerlos en cuenta como aspecto clave para el desarrollo de intervenciones preventivas, psicoterapéuticas y farmacológicas (Hartwell, Back, McRaeClark, Shaftman y Brady, 2012). Dado que las experiencias relacionadas con el consumo de sustancias durante la adolescencia afectan y pueden producir cambios en las expectativas de consumo en etapas más tardías (Monk y Heim, 2016), comprender las razones de uso del cannabis en esta etapa puede ser un factor importante para el desarrollo de intervenciones (Fox, Towe, Stephens, Walker y Roffman, 2011). Además, dicha evaluación se debe realizar de forma específica para cada sustancia, ya que se han observado diferentes motivos de consumo para las diferentes sustancias (Hartwell, Back, McRae-Clark, Shaftman y Brady, 2012), así como diferentes motivos de consumo en función del trastornos mentales comórbidos que presentan los pacientes (Thornton et al., 2012). Por estos motivos, la existencia de instrumentos de medida, específicos para población adolescente consumidora de cannabis, que evalúan la intención de consumo (Lloret Irles, Morell-Gomis, Laguía y Moriano, 2018), la motivación al cambio (Kaminer, Ohannessian, McKay y Burke, 2016) o los motivos de consumo, son de gran ayuda para la planificación y monitorización 
de las intervenciones clínicas. Sin embargo, la investigación sobre cuáles son los motivos de consumo de cannabis en población adolescente que presenta un trastorno mental es escasa y no existe ningún estudio en nuestro entorno, siendo una de las razones de la falta de estudios la no existencia instrumentos validados en español para medir los motivos de consumo.

El objetivo del presente trabajo es estudiar las propiedades psicométricas del MMM en una muestra de adolescentes con trastorno por uso de cannabis. Se intenta así subsanar el vacío existente entre los instrumentos de medida con buenas propiedades psicométricas específicos para cada sustancia. Los objetivos específicos del estudio son realizar la traducción y adaptación del cuestionario MMM de Simons, Correia, Carey y Borsari (1998) y analizar la estructura, fiabilidad y validez de las puntuaciones de la versión española del cuestionario MMM. Se espera que la versión española del MMM muestre una estructura factorial similar a la versión original ( Simons, Correia, Carey y Borsari, 1998), una buena fiabilidad de la puntuación total del instrumento y de las puntuaciones de cada una de las subescalas. También se espera que los índices encontrados no muestren gran diferencia a la validación llevada a cabo en población adolescente francesa Chabrol, Ducongé, Casas, Roura y Carey, 2005).

\section{Método}

\section{Participantes}

Los participantes fueron seleccionados durante los años 2011-2014 a través de muestreo consecutivo entre los usuarios de la Unidad de Conductas Adictivas del Adolescente del Servicio de Psiquiatría del Hospital de Sant Joan de Déu, Barcelona. La muestra estuvo formada por 228 participantes, $64.9 \%(\mathrm{n}=148)$ chicos y el $35.1 \%(\mathrm{n}=80)$ chicas, con una edad media de 15.7 años $(\mathrm{DE}=1.3)$.

\section{Instrumentos}

Registro del consumo: el consumo de cannabis se evaluó a partir de la administración de una entrevista clínica ad hoc registrando, entre otros, los siguientes datos: Edad de inicio del consumo (primer consumo) y edad de consumo regular (desde 1 consumo mensual); consumo actual (últimos 6 meses y último mes)

Psicopatología: se utilizó la entrevista semi-estructurada Kiddie-Sads-Present and Lifetime (K-SADS-PL), la versión española (Mattos y Rohde, 2007), que sigue los criterios DSM-IV. La K-SADS-PL es una entrevista diagnóstica semiestructurada diseñada para evaluar los episodios pasados y actuales de psicopatología en niños y adolescentes, siguiendo los criterios DSM-IV. Esta escala es administrada a los padres y al propio paciente por separado, obteniendo una puntuación final que recoge todas las fuentes de información utilizadas.

Motivos de consumo: se utilizó la traducción española del Marihuana Motive Measure (MMM) (Simons, Correia, Carey y Borsari, 1998). Cuestionario de 25 ítems con una respuesta tipo likert. En población adulta, el estudio de la estructura factorial ha mostrado 5 factores (Expansion, Social, Conformity, Enhancementy y Coping) y ha mostrado que su medida es fiable y válida (ver introducción).

Efectos subjetivos del consumo de cánnabis: Se administró el Addiction Research Center Inventory (ARCI) (Martin, Sloan, Sapira y Jasinski, 1971), la versión validada en población española (Lamas, Farré, Llorente, y Camí, 1994). Este cuestionario consta de 49 ítems y de 5 escalas: La escala Pentobarbital-Chlorpromazine-Alcohol Group (PCAG), que puntúa la intensidad del efecto de 0 a 15, y mide sedación. La escala Morphine-Benzedrine Group (MBG) que puntúa de 0 a 16 y que mide la euforia. La escala Lysergic-acid-Dyethyl-amide Scale (LSD), que puntúa de 0 a 14 y mide disforia y síntomas de tipo somático. La escala Benzedrine Group (BG), que puntúa de 0 a 13 y mide estimulación. La escala Amphetamine (A), que puntúa de 0 a 11 y mide fenómenos empíricos (Busquets, Torrens, Soler, Farré i Bulbena, 2005). En la versión validada en población española (Lamas, Farré, Llorente y Camí, 1994), los coeficientes de fiabilidad de las escalas son: PCAG 0,87; MBG 0,81; LSD 0,55; BG 0,79; y A 0,49 .

Personalidad: Se administró el Millon Adolescent Clinical Inventroy (MACI) (Millon, 1993). Consta de 160 ítems con formato verdadero/falso y está organizado en 31 escalas, 27 con significado clínico, 12 de patrones de personalidad, 8 de preocupaciones expresadas y 7 de síntomas clínicos. En la versión validada en población española, los coeficientes alfa de las escalas básicas de personalidad varían de 0.74 (sumiso) a 0.90 (autopunitivo) (Millon, 2004).

\section{Procedimiento}

La traducción del cuestionario se realizó siguiendo las directrices para la traducción y adaptación de test de la Comisión Internacional de Test (Muñiz, Elosua y Hambleton, 2015). Tras valorar la relevancia del constructo y del cuestionario, se contactó con el autor (Dr. Simons) que dio su autorización para realizar el estudio del MMM. Posteriormente se realizaron dos traducciones independientes. Por un lado los autores del estudio realizaron conjuntamente una traducción prestando especial interés a las características lingüísticas, psicológicas y culturales del texto adaptado. Por otro lado, el cuestionario fue traducido de forma independiente por tres profesionales hispanohablantes expertos en salud mental infanto-juvenil y con elevado nivel de inglés. Una vez se realizaron las diferentes traducciones, estas fueron comparadas, discutidas las diferencias y consensuado el texto final en una reunión en la que participaron los diferentes traductores. En esta fase, el consenso se 
buscó teniendo en cuenta tanto los aspectos lingüísticos, como consideraciones conceptuales y culturales. Posteriormente una traductora oficial realizó la retrotraducción, que fue enviada al Dr. Simons. Una vez las modificaciones propuestas por el autor original del cuestionario fueron consideradas por los autores, se realizaron las modificaciones oportunas y se aprobó la traducción. Esta traducción fue pasada a 15 pacientes de la Unidad de Conductas Adictivas del Adolescente en entrevistas independientes, en la que se valoró la comprensión del contenido del cuestionario. Los resultados de estas últimas entrevistas se comentaron finalmente entre los autores y se realizaron los cambios por consenso. La versión final fue nuevamente revisada por la traductora oficial y aprobados por todos los profesionales que participaron en la traducción.

Una vez se obtuvo la aprobación del Comité de Ética del Hospital Sant Joan de Déu se inició el estudio. Antes de ser incluidos en el estudio, los participantes eran informados sobre el mismo y si aceptaban participar firmaban el consentimiento. Los criterios de inclusión fueron tener una edad inferior a 18 años y presentar criterios de abuso o dependencia (DSM-IV-TR) en el momento de la evaluación. Como único criterio de exclusión se consideró presentar patología mental aguda que dificultase la comprensión de los cuestionarios. Ningún participante fue excluido por este criterio.

La evaluación realizada es parte del protocolo diagnóstico habitual de la Unidad de Conductas Adictivas del Adolescente, en el que se realizan en tres visitas de 45 minutos de duración aproximada. En la primera se realiza la anamnesis psiquiátrica y toxicológica, el registro de consumo y la analítica de orina. En la segunda, se administra la entrevista Kiddie-Sads a los progenitores o tutores y se entregaban los cuestionarios al adolescente. En la tercera visita, se recogen los cuestionarios y se supervisaba que estuviesen debidamente cumplimentados, evitando de esta forma los valores perdidos. 24 pacientes no completaron correctamente todos los cuestionarios motivo por el que no fueron incluidos en el estudio.

\section{Análisis estadístico}

El estudio de las propiedades psicométricas del MMM se realizó siguiendo los siguientes pasos, estudio de la estructura factorial, de la fiabilidad de las medidas del mismo y de la evidencia de validez. El software estadístico utilizado fue el SPSS 21 (IBM Corp, 2010). La estructura factorial del MMM se estudió utilizando un análisis factorial de componentes principales. Como se esperaba que las diferentes subescalas correlacionaran entre ellas (Simons, Correia, Carey y Borsari, 1998), se realizó un procedimiento de componentes principales y rotación promax. El nombre de factores retenidos se determinó en función del análisis de dos criterios. El primero fue el de los autovalores de los factores extraídos, y el segundo a partir del estudio del cambio de pendiente en el gráfico de sedimentación delos diferentes factores. Una vez determinado el número de factores, se estudió la fiabilidad de las diferentes medidas del cuestionario a partir del Alfa de Cronbach. Igualmente se analizó la fiabilidad test-retest de la medida en una submuestra del total $(n=24)$ a partir de correlaciones intraclase. El estudio de la evidencia de validez de las medidas del MMM se realizó a partir de correlaciones de Pearson entre las medidas de las subescalas del MMM y la de los cuestionarios MACI y ARCI.

\section{Resultados}

\section{Descripción de la muestra}

La edad media de inicio del consumo fue de 12.9 años $(D E=1.7)$ y la edad media de consumo regular de 13.8 años $(D E=1.5)$. No se observan diferencias significativas en la distribución por sexos en edad de inicio de consumo (chicos: media $=13,07 ; D E=1,69$ vs. chicas: media $=12,81$; $\left.D E=1,6 ; t_{(226)}=1,14 ; p=0,25\right)$, ni en edad de consumo regular (chicos: media $=13,86 ; D E=1,44$ vs. chicas: media $=$ 13,$\left.59 ; D E=1,59 ; t_{(226)}=1,34 ; p=0,19\right)$.

El $83,8 \%$ de los adolescentes manifestaron preferir el consumo de marihuana $(n=191)$ al consumo de hachís, sin observar diferencias por sexo (chicos: 81,8\%; $(n=121)$ vs. chicas: $\left.87,5 \%(n=70) ; \chi_{(1)}^{2}=1,26 ; p=0,26\right)$. Los participantes fueron diagnosticados siguiendo los criterios del DSM IV-TR, el $32.9 \%(n=75)$ fue diagnosticado de abuso de cánnabis y el $67.1 \%(n=153)$ de dependencia de cánnabis, sin diferencias significativas por sexo $\left(\chi_{(1)}^{2}=0,009\right.$; $p=0,93)$.

Respecto a la psicopatología comorbida, se observó que el $62,7 \%(n=143)$ presentaba un trastorno de conducta (síndrome que engloba el Trastorno del Comportamiento No Especificado, el Trastorno Negativista Desafiante y el Trastorno Disocial), el 11,8\% $(n=27)$ presentaba un Trastorno por Déficit de Atención e Hiperactividad, el 11,8\% $(n=27)$ un Trastorno Psicótico, el 11,0\% $(n=25)$ un trastorno afectivo (agrupando Trastorno Depresivo Mayor y Trastorno Bipolar), el 3,1\% $(n=7)$ un Trastorno Adaptativo, el 3,9\% $(n=9)$ un Trastorno de la Conducta Alimentaria y el $4,4 \%(n=10)$ un trastorno de ansiedad.

\section{Estructura factorial del $\mathbf{M M M}$}

El análisis factorial de componentes principales realizado mostró una estructura de cinco factores con valor propio igual o superior a 1,00. La estructura resultante explica una varianza del 53,8\%. El análisis factorial mostró un índice de Kaiser-Meyer-Olkin de 0,82 y una prueba de esfericidad de Bartlett significativa $\left(\chi^{2}=1917,02\right.$; g.l. $=300$; $p<0,001)$; resultado que indica una selección adecuada del procedimiento para reducir la escala. La solución de cinco factores es igual que la escala original a excepción de los ítems 15 y 5 (ver Tabla 1); respecto estos ítems, nuestros re- 
sultados muestran que el ítem 15 ("Porqué me siento más confiado y seguro de mí mismo") carga en el factor Coping en lugar de en el de Social y el ítem 5 ("ser sociable") tenía una carga similar sobre todos los factores (ver Tabla 1).

Tabla 1. Análisis factorial exploratorio del MMM

\begin{tabular}{|c|c|c|c|c|c|}
\hline Ítems & Coping & Expansion & Social & Enhancement & Conformity \\
\hline (1) Para olvidar mis preocupaciones & 89 & &,- 21 & & \\
\hline (17) Para olvidar los problemas & ,89 & &,- 11 & & \\
\hline (6) Para animarme cuando estoy de mal humor & 65 & &, 22 & & \\
\hline (15) Porque me siento más seguro y con más confianza & ,43 & ,35 & & & \\
\hline (23) Para entender las cosas de forma diferente & & 81 & & &,- 13 \\
\hline (24) Para ampliar los límites de la mi conciencia & & ,76 & & &,- 23 \\
\hline (22) Porque me ayuda a ser más creativo y original & & 67 &,- 17 & ,21 & \\
\hline (25) Para estar más abierto a nuevas experiencias & &, 57 & & & \\
\hline (16) Para celebrar las ocasiones especiales con los amigos & & & ,86 & & \\
\hline (14) Porque hace más divertidas las celebraciones & & & 84 & & \\
\hline (3) Porque me ayuda a divertirme & & &, 77 & & \\
\hline (11) Porque hace que los acontecimientos sociales sean más divertidos & & &, 70 & 15 & \\
\hline (7) Porque me gusta la sensación & & & & ,79 &,- 19 \\
\hline (13) Porque me provoca placer & & & &, 70 & 16 \\
\hline (18) Porque es divertido & & & ,23 &, 54 & 10 \\
\hline (9) Porque es emocionante & & & & ,45 & \\
\hline (20) Para no sentirme aislado delos demás & &,- 12 &,- 15 & & 80 \\
\hline (12) Para sentir que formo parte del grupo de gente que me gusta & & & & & ,79 \\
\hline (19) Para gustar a los demás & & &,- 12 & ,13 & ,73 \\
\hline (2) Porque mis amigos me insisten &,- 14 & ,34 & &,- 21 &, 43 \\
\hline (8) Porque los otros no reirían de mí por no hacerlo & &,- 22 & ,20 &,- 13 & 35 \\
\hline (5) Para ser sociable &, 23 &, 23 &, 21 &,- 12 &,- 28 \\
\hline Autovalor & 6,09 & 2,38 & 1,97 & 1,84 & 1,17 \\
\hline$\%$ variancia & 24,34 & 9,51 & 7,89 & 7,36 & 4,68 \\
\hline Alfa de Cronbach & ,83 & ,74 & ,82 & ,72 & 65 \\
\hline
\end{tabular}

Nota. Los valores inferiores a o,10 no se incluyeron a la tabla.

Matriz rotada promax.

\section{Fiabilidad de las medidas del MMM}

El estudio de la fiabilidad mostró una fiabilidad alta de la puntuación total del MMM (Alfa de Cronbach=0,86) y valores entre altos y moderados en las puntuaciones de cada una de las subescalas (Social $=0,82$; Enhancement $=$ 0,72; Coping $=0,83 ;$ Expansion $=0,74 ;$ Conformity $=0,64)$, como se puede observar en la Tabla 1. Las correlaciones de Pearson de las puntuaciones de las subescalas oscilaron entre 0,21 entre las puntuaciones de Conformity y Expansion y 0,57 entre las puntuaciones de Enhancement y Social (en todos los casos $p=0,01$ ).

La correlación intraclase entre el test-retest de las diferentes puntuaciones del MMM indicó correlaciones iguales o inferiores a 0,42 . Las correlaciones fueron significativas para todas las puntuaciones $($ Coping $=0,40 ;$ Enhancement $=$ 0,35; Expansion = 0,30 y MMM total = 0,42; todas las $p \leq 0,05)$, con las excepción de Social $(0,22 ; p=0,12)$ y Conformity $(0,001)$.

\section{Evidencia de validez de las medidas del MMM}

La evidencia de validez del MMM se estudió a partir de las correlaciones entre el uso actual de cánnabis, el cuestio- nario de efectos subjetivos del consumo (ARCI) y el cuestionario de personalidad (MACI). El consumo de cánnabis en los últimos 6 meses tubo una asociación significativa y positiva con el motivo de Coping ( $r=0,22, p=0,001)$ y el motivo de Enhancement ( $r=0,21, p=0,002$ ). No obstante, el uso de cannabis no se asoció significativamente con el motivo de Expansion $(r=0,11, p=0,09)$, Social $(r=0,08, p=0,220)$, o el de Conformity $(r=-0,06, p=0,359)$. Como se puede observar en la Tabla 2, los efectos subjetivos del cánnabis mostraron correlaciones significativas con los motivos de consumo. La escala de Coping del MMM correlacionó significativamente con todas las escalas del ARCI $(r=$ de 0,24 hasta 0,$35 ; p \leq$ $0,01)$, a excepción de la escala de eficiencia intelectual. La escala Expansion correlacionó significativamente con la de euforia $(p<0,001)$ y la eficiencia intelectual $(p=0,006)$ del ARCI. La escala Social correlacionó significativamente con euforia $(p=0,002)$ y disforia/síntomas somáticos del ARCI $(p=0,03)$. La escala Enhancement sólo correlaciona significativamente con la escala de euforia del ARCI $(p=0,001)$. Finalmente, la escala Conformity correlacionó significativamente con los síntomas empíricos $(p=0,001)$, euforia ( $p$ $=0,02)$ y sedación $(p=0,01)$. Finalmente, las correlacio- 
nes de las 5 escalas del MMM con las de personalidad del MACI, mostraron que las correlaciones significativas se observaron principalmente con las escalas de Coping y Conformity del MMM (Ver Tabla 2), observándose correlaciones positivas de Copingy Conformity con casi todas las escalas del
MACI, con excepción de las escalas histriónico $(-0,29 ; p=$ $0,01)$, egocéntrico $(-0,26 ; p=0,01)$ y conformista $(-0,27 ; p$ $=0,01)$ que correlacionaban negativamente con Coping; $\mathrm{e}$ histriónico $(-0,27 ; p=0,01)$ y egocéntrico $(-0,24 ; p=0,01)$ con correlaciones negativas con Conformity (ver Tabla 2).

Tabla 2. Validez del MMM utilizando la correlación de Pearson con los cuestionarios ARCI y MACI

\begin{tabular}{|c|c|c|c|c|c|}
\hline & \multicolumn{5}{|c|}{ Factores ММM } \\
\hline & Coping & Expansion & Social & Enhancement & Conformity \\
\hline \multicolumn{6}{|l|}{$\mathrm{ARCl}$} \\
\hline Sedación &, $26 * \star$ &, 12 & ,11 &, 07 &, $17^{\star}$ \\
\hline Euforia &, $35^{\star \star}$ &, $39 * \star$ &, $24^{\star \star}$ &, $22 * \star$ &, $16^{\star}$ \\
\hline Disforia & $24^{\star \star}$ & ,09 & $15^{\star}$ & ,12 & ,11 \\
\hline Estimulación & ,08 &, $18^{\star \star}$ &,- 03 & ,01 &,- 007 \\
\hline Fenómenos empíricos &, $28^{\star \star}$ &, $29 * \star$ &, 08 &, 007 &, $22^{\star \star}$ \\
\hline \multicolumn{6}{|l|}{$\mathrm{MACl}$} \\
\hline Introvertido &, $21 * \star$ & ,08 &,- 01 &,- 06 &, $23 * \star$ \\
\hline Inhibido &, $21 * \star$ & 01 & 02 &,- 05 & $29 * \star$ \\
\hline Pesimista &, $38^{\star \star}$ & ,15 &,- 07 &,- 07 &, $21 * \star$ \\
\hline Sumiso & ,12 &,- 09 & ,03 &,- 08 &, $21 * \star$ \\
\hline Histriónico &,$- 27 * \star$ & 01 & ,03 & 04 &,$- 27^{\star \star}$ \\
\hline Egocéntrico &,$- 26 * \star$ & 04 &, 004 & 07 &,$- 24^{\star \star}$ \\
\hline Rebelde &,- 004 & ,14 &, 02 & ,14 &,- 15 \\
\hline Rudo &, 06 & 07 &,- 001 & ,13 &,- 05 \\
\hline Conformista &,$- 27 * \star$ &,$- 22^{\star \star}$ &,- 004 &,- 10 &,- 06 \\
\hline Oposicionista &, $29 * \star$ &, $21 * \star$ &,- 01 &,- 01 &, 04 \\
\hline Autopunitivo &, $33^{\star *}$ &, 12 &,- 05 &,- 002 &, $24^{\star \star}$ \\
\hline Tendencia Límite &, $29 * \star$ & ,008 & ,002 & ,003 & ,11 \\
\hline
\end{tabular}

Nota. $\mathrm{MMM}=$ Marihuana Motives Measure; $\mathrm{ARCl}=$ Addiction Research Center Inventory; $\mathrm{MACl}=$ Millon Adolescent Clinical Inventory. ${ }^{\star \star}: \mathrm{p}=0,01 ;{ }^{\star}: \mathrm{p}=0,05$.

\section{Discusión}

Este estudio se realizó para analizar de las propiedades psicométricas de la versión española del Marihuana Motive Measure (MMM) en una muestra de adolescentes con trastorno por uso de cannabis. Como se esperaba, el análisis factorial del cuestionario muestra una estructura factorial de 5 factores, similar a la propuesta por el autor original. Además, los resultados obtenidos de fiabilidad y evidencia de validez de las diferentes medidas de la versión española del MMM indican que este es un buen instrumento para ser utilizado con adolescentes españoles consumidores de cannabis.

La versión española del MMM mostró la misma estructura factorial que la versión original (Simons, Correia, Carey y Borsari, 1998). La solución de cinco factores (Social, Coping, Conformity, Enhancement y Expansion) obtenida es igual a la estructura de la escala original a excepción de los ítems 15 y 5. El ítem 15 ("Porque me siento más confiado y seguro de mí mismo) carga en el factor Coping en lugar de en el de Social. Dicha carga del ítem 15 en el factor Coping puede ser explicada por la percepción de los pacientes de que el consumo les ayuda a afrontar las situaciones o emociones complicadas con más seguridad y confianza. Por otro lado, la carga del ítem 5 ("Ser sociable") era similar sobre todos los factores principalmente el de Coping, Expansion y Social, y podría ser interpretada como la percepción de los pacientes de que el consumo les provocaba una mayor desinhibición social y por tanto, en algunos de ellos, les ayudaría a afrontar mejor aquellas situaciones sociales en las que se pueden encontrar más ansiosos o inhibidos. Este hecho también explicaría la carga negativa de estos ítems en el factor Conformity.

La consistencia interna es buena para el total y para cada una de las escalas a excepción de la de Conformity que muestra una consistencia interna moderada. Una posible explicación se basa en la dificultad de los adolescentes en reconocer el miedo a ser rechazado. Es decir, cuando un adolescente se encuentra en un contexto de amigos consumidores y no dispone de las habilidades suficientes para gestionarlo, estos ejercen una presión directa o social para consumir. Por otro lado, esta consistencia moderada puede ser debida a que el factor Conformity incluye ítems relacionados con el deseo o la necesidad de sentirse reconocido o de aprobación grupal e ítems relacionados con el miedo al rechazo. El deseo o la necesidad de aprobación y el miedo al rechazo pueden no estar presentes en todos los pacientes, o ellos pueden no ser conscientes de la relación entre estos dos motivos y su conducta gregaria. Finalmente, otra explicación de esta consistencia moderada podría ser que las herramientas diseñadas para población adulta no 
se han de comportar necesariamente de la misma manera cuando se aplican a población adolescente. La diferencia en el desarrollo, los patrones de consumo la relación con la familia y los compañeros puede afectar a la estructura factorial de los instrumentos (Martin, Copeland, Gilmour, Gates, Swift, 2006). No obstante, se puede considerar que la estructura factorial de la traducción al español muestra una buena validez de contenido para las diferentes escalas del cuestionario.

En relación a la fiabilidad test-retest de las puntuaciones, se esperaba que las correlaciones encontradas fueran bajas entre las dos medidas realizadas con el MMM. Esta hipótesis se elaboró debido a que el MMM es un cuestionario que evalúa los motivos de consumo en un determinado momento y, según el modelo motivacional, estos son susceptibles de modificarse en función del proceso de cambio que viva el paciente. En este sentido, los resultados observados en el presente trabajo muestran una correlación intraclase baja (Fleiss, 1986), lo que indicaría, tal y como se ha comentado, que los motivos de consumo se ven modificados a partir de la intervención psicoterapéutica. La única excepción fue observada con la correlación intraclase para la escala de Coping. Esto podría estar indicando la necesidad que pueden presentar los pacientes de consumir para aliviar el malestar y lo que se denomina la "espiral del malestar", es decir, el mantenimiento del consumo ya no para pasarlo bien sino para no sufrir, y que sería un indicador de severidad del consumo. Esta afirmación gana consistencia si se tiene en cuenta que la muestra del estudio era de pacientes que presentaban trastorno por uso de cannabis con una la alta prevalencia de trastornos mentales comorbidos. Los resultados obtenidos plantean la posibilidad de que el MMM sea una herramienta adecuada para la monitorización del cambio en el proceso terapéutico.

La asociación observada entre los motivos y los efectos subjetivos del consumo es significativa y en la misma dirección, tal y como se observa en otros estudios (Deker, Linszen y De Haan, 2009). En este sentido, los resultados del presente estudio muestran que los motivos de Coping están relacionados con las escalas de sedación y disforia, relación que puede ser entendida como la necesidad del paciente a buscar la regulación del afecto y la relajación. Del mismo modo, la relación positiva entre Copingy el efecto subjetivo de euforia, puede estar indicando el aumento de la energía secundario a la disminución del malestar. El factor de Conformity se relaciona con la escala de sedación, indicando que los que consumen por Conformity pueden hacerlo buscando disminuir la desazón o la preocupación cuando están expuestos a contexto interpersonal. Finalmente, la relación observada entre los motivos de consumo de Expansion con la escala de fenómenos empíricos es directa al estar evaluando los dos cuestionarios el mismo constructo, aunque la relación no es perfecta. Esta relación moderada de dos fenómenos puede ser explicada porque el factor de Expansion mide motivación y la escala de fenómenos empíricos, síntomas.

La intensidad de la relación entre los motivos del MMM y las escalas del ARCI concuerdan con el estudio de Dekker, Linszen y De Haan (2009) donde los consumidores de cannabis explicaban muchos más efectos positivos que negativos en la modulación del afecto y el aumento de la relajación con el consumo y, moderadamente, más efectos positivos que negativos en el aumento de la energía y la socialización. De hecho, los autores afirman que los efectos autoreportados del consumo se podrían agrupar en 4 grupos principales: mejorar las sensaciones positivas, aliviar la disforia, razones sociales y razones relacionadas con la enfermedad y los efectos sedativos de la medicación.

Estos resultados son importantes ya que aportan más peso a la necesidad de abrir líneas futuras de investigación que ayuden a aclarar la relación entre la respuesta subjetiva al consumo de drogas como importante predictor del posterior consumo y abuso de esta sustancia, tal y como se ha apuntado en estudios previos (Littlefield, Sher y Wood, 2010a; Adams, Kaiser, Lynam, Charnigo y Milich, 2012). En este sentido, dichas investigaciones señalan que las experiencias de consumo positivas se asocian a un uso más prolongado y a un incremento del riesgo de abuso y dependencia, mientras que las negativas se asocian a un menor tiempo y menor frecuencia de consumo (Scherrer et al., 2009; Zeiger et al., 2012).

El estudio de la relación entre los motivos de consumo y la personalidad se fundamenta en el papel mediador que tienen los motivos entre la personalidad y el consumo (Littlefield, Sher y Wood, 2010a; Adams, Kaiser, Lynam, Charnigo y Milich, 2012) y, también, en entender la conceptualización de que los rasgos de personalidad contribuyen a la motivación de la conducta en general y a las motivaciones para el consumo de sustancias, en particular (Littlefield, Sher y Wood, 2010b). Nuestros resultados observan una relación entre los motivos para Copingy los rasgos de personalidad relacionados con la inseguridad y con la presencia de dificultades en la esfera interpersonal. También, tal y como se esperaba, los motivos de Conformity se relacionan con las personalidades sumisas y con dificultades en la asertividad. Estos resultados son consistentes con otros estudios donde se ha observado una clara y directa asociación entre el neuroticismo y el motivo por Coping (Kuntsche, Knibbe, Gmel y Engels, 2006). Por otra parte, la investigación previa había relacionado la impulsividad con el motivo de Enhancement (Littlefiel, Sher y Wood, 2010), argumentando que este motivo actúa como mediador entre la extraversión y el consumo de alcohol (Adams, Kaiser, Lynam, Charnigo y Milich, 2012). Sin embargo, no hemos podido confirmar estos resultados en nuestro estudio al no relacionarse significativamente las escalas básicas de personalidad con el motivo por Enhancement. Este resultado contradictorio podría explicarse por el tipo de cuestionario utilizado en este 
trabajo, pensado para evaluar características de personalidad patológica, y por el hecho de que éste fue aplicado a una muestra de pacientes que estaban en tratamiento por problemas relacionados con el consumo de cannabis.

A pesar del interés de los resultados del estudio, este muestra ciertas limitaciones que tienen que ser tenidas en cuenta a la hora de generalizar los resultados y que ponen de relieve la necesidad de estudios que confirmen las propiedades psicométricas del MMM en otras muestras. En primer lugar, la muestra estuvo formada por adolescentes que acudieron en busca de tratamiento para el trastorno por uso de cannabis, por este motivo, la generalización de los resultados a muestras de la comunidad de adolescentes con patrones de consumo más normativos debe tomarse con cautela. Sin embargo, tal y como se comentó en la introducción, el presente estudio es el primero que se realiza en pacientes que presentan trastorno por consumo de cannabis. En este sentido, los resultados del presente trabajo muestran que las medidas del MMM de los motivos de consumo, cuando es utilizado en adolescentes con trastorno por consumo de cannabis, muestra propiedades psicométricas similares a las obtenidas por el autor original (Simon et al., 1998) y en población adolescente francesa (Chabrol, Ducongé, Casas, Roura y Carey, 2005), poniendo de relieve la utilidad del MMM en esta población y la necesidad de futuros estudios que incluyan adolescentes con consumo más normativo. En segundo lugar, el procedimiento utilizado para el estudio de la fiabilidad no ha sido el más apropiado (Zumbo et al 2007). Aunque el coeficiente de consistencia alfa de Cronbach ha sido ampliamente utilizado y aún se utiliza como índice de consistencia interna en los cuestionarios que utilizan escala de respuesta tipo Likert, el uso este índice puede ser una estimación atenuada del límite inferior de la fiabilidad, especialmente cuando los ítems tienen pocas opciones de respuesta y la asimetría de los mismo es alta. En estos casos el coeficiente alfa ordinal es más adecuado (Gadermann, Guhn y Zumbo, 2012). Aunque el número de respuesta del MMM es de 5 y la asimetría de las respuesta a los ítems se aproxima a 0 en la mayoría de los ítems, lo que no permite inferir una atenuación baja del alfa de Cronbach (Gadermann, Guhn y Zumbo 2012) y la estimación realizada en el presente trabajo nos permite compararla con estudios previos (Simon et al., 1998, Chabrol, Ducongé, Casas, Roura y Carey, 2005), estudios futuros deberán confirmar la fiabilidad de las medidas utilizando el coeficiente alfa ordinal. En tercer lugar, la falta de herramientas validadas en nuestro contexto para valorar de forma más consistente la evidencia de validez del instrumento hizo necesario el estudio de ésta a partir del uso de medidas de constructos relacionados de forma indirecta. A pesar de estas limitaciones, consideramos que los resultados del presente trabajo son de gran relevancia, ya que se centra en la validación de una herramienta que nos permitirá desarrollar futuras investigaciones en un campo tan importante como es el abuso de substancias en la adolescencia.

En conclusión, dado que desde un punto de vista terapéutico, actuar de forma precoz en los adolescentes consumidores de cannabis es un elemento clave para prevenir las consecuencias negativas a larga plazo (Castro-Fornieles, 2013) asociadas al consumo. La comprensión de los motivos para iniciar y mantener el consumo puede ser útil para identificar a aquellos adolescentes que estén en situación de riesgo y para el establecimiento de programas de prevención e intervención (Lee, Neighbors, Hendershot, Greossbars, 2009). Los resultados de este estudio, realizado en una muestra clínica, muestran que las medidas de los motivos de consumo obtenidas con el MMM hacen de este instrumento una herramienta útil para el estudio y en la práctica clínica con estos pacientes. Contar con instrumentos de medidas como el MMM aplicables en nuestro entorno posibilitan la evaluación individual, para poder abordar la complejidad de las razones por las que los adolescentes consumen drogas y abre la oportunidad de futuros estudios dada la importancia de los aspectos motivacionales para mejorar la comprensión y el manejo de estos adolescentes (Miller y Rollnick, 1991).

\section{Conflicto de intereses.}

Los autores declaran no tener ningún conflicto de interés.

\section{Referencias}

Adams, Z.W., Kaiser, A.J., Lynam, D.R., Charnigo, R.J. y Milich, R. (2012). Drinking motives as mediators of the impulsivity-substance use relation: pathways for negative urgency, lack of premeditation, and sensation seeking. Addictive Behaviors, 37, 848-55. doi:10.1016/j. addbeh.2012.03.016.

Arias Constantí, V., Sanz Marcos, N., Trenchs Sainz de La Maza, V., Curcoy Barcenilla, A.I., Matalí Costa, J. y Luaces Cubells, C. (2010). Psychoactive drugs use and related visits of adolescents to the emergency department. Medicina Clínica (Barcelona), 134, 583-586. doi:10.1016/j. medcli.2010.01.019.

Busquets, E., Torrens, M., Soler, A., Farré, M. y Bulbena, A. (2005). Differences in the subjective effects of drugs in patients with a first psychotic episode. Preliminary results. Actas Españolas de Psiquiatría, 33, 19-25.

Castro-Fornieles, J. (2013). Child and adolescent psychiatry: the need for training and development. Revista de Psiquiatría y Salud Mental, 6, 57-59. doi:10.1016/j. rpsm.2012.10.001.

Chabrol, H., Ducongé, E., Casas, C., Roura, C. y Carey, K.B. (2005). Relations between cannabis use and dependence, motives for cannabis use and anxious, depressive 
and borderline symptomatology. Addictive Behaviors, 30, 829-840. doi:10.1016/j.addbeh.2004.08.027.

Cooper, L. (1994). Motivations for alcohol use among adolescents: Development and validation of a four-factor model. Psychological Assessment, 6, 117-128. doi:10.1037/ 1040-3590.6.2.117.

Degenhardt, L., Coffey, C., Carlin, J.B., Swift, W., Moore, E. y Patton, G.C. (2010). Outcomes of occasional cannabis use in adolescence: 10-year follow-up study in Victoria, Australia. The British Journal of Psychiatry, 196, 290-295. doi:10.1192/bjp.bp.108.056952.

Dekker, N., Linszen, D.H. y De Haan, L. (2009). Reasons for cannabis use and effects of cannabis use as reported by patients with psychotic disorders. Psychopathology, 42, 350-360. doi:10.1159/000236906.

Fleiss, J.L. (1986). The design and analysis of clinical experiments. Nueva York: John Wiley \& Sons, Inc.

Fox, C.L., Towe, S.L., Stephens, R.S., Walker, D.D. y Roffman, R. (2011). Motives for cannabis use in high-risk adolescent users. Psychology of Addictive Behaviors, 25, 492-500. doi:10.1037/a0024331.

Gadermann, A.M., Guhn, M. y Zumbo, B.D. (2012). Estimating ordinal reliability for Likert-type and ordinal item response data: A conceptual, empirical, and practical guide. Practical Assessment, Research E Evaluation, 17, 1-13. Recuperado de http://pareonline.net/pdf/ v17n2.pdf.

Hartwell, K.J., Back, S.E., McRae-Clark, A.L., Shaftman, S.R. y Brady, K. T. (2012). Motives for using: a comparison of prescription opioid, marijuana and cocaine dependent individuals. Addictive Behaviors, 37, 373-378. doi:10.1016/j.addbeh.2011.11.014.

Horwood, L.J., Fergusson, D.M., Hayatbakhsh, M.R., Najman, J.M., Coffey, C., Patton, G. C.,... Hutchinson, D.M. (2010). Cannabis use and educational achievement: findings from three Australasian cohort studies. Drug and Alcohol Dependence, 110, 247-253. doi:10.1016/j.drugalcdep.2010.03.008.

IBM Corp. Released 2010. IBM SPSS Statistics for Windows, Version 19.0. Armonk, NY: IBM Corp.

Kuntsche, E., Knibbe, R., Gmel, G. y Engels, R. (2006) Who drinks and why? A review of socio-demographic, personality and contextual issues behind the drinking motives in young people. Addictive Behaviors, 31, 1844-1857. doi:10.1016/j.addbeh.2005.12.028.

Kaminer, Y., Ohannessian, C.M., McKay, J.R. y Burke, R.H. (2016). The Adolescent Substance Abuse Goal Commitment (ASAGC) Questionnaire: An Examination of Clinical Utility and Psychometric Properties. Journal of Substance Abuse Treatment, 61, 42-46. doi:10.1016/j. jsat.2015.09.007.

Lamas, X., Farré, M., Llorente, M. y Camí, J. (1994). Spanish version of the 49-item short form of the Addiction Re- search Center Inventory (ARCI). Drug and Alcohol Dependence, 35, 203-209. doi:10.1016/0376-8716(94)90075-2.

Littlefield, A.K., Sher, K.J. y Wood, P.K. (2010a). Do changes in drinking motives mediate the relation between personality change and "maturing out" of problem drinking? Journal of Abnormal Psychology, 119, 93-105. doi:10.1037/a0017512.

Littlefield, A., Sher, K. y Wood, P. (2010b). A Personality-Based Description of Maturing Out of Alcohol Problems: Extension with a Five-Factor Model and Robustness to Modeling Challenges. Addictive Behaviors, 35, 948-954. doi:10.1016/j.addbeh.2010.06.008.

Lee, C.M., Neighbors, C., Hendershot, C.S. y Grossbard, J.R. (2009). Development and preliminary validation of a comprehensive marijuana motives questionnaire. Journal of Studies on Alcohol and Drugs, 70, 279-287. doi:10.15288/jsad.2009.70.279.

Lloret Irles. D., Morell-Gomis, R. Laguía, A. y Moriano, J.A. (2018). Diseño y validación de una escala de intención de consumo de cannabis (CUIQ) para adolescentes. Adicciones, 30, 54-65. doi:10.20882/adicciones.865.

Mattos, P. y Rohde, L.A. (2007). The Kiddie-Sads Present and Lifetime Version. Journal of Attention Disorders, 11, 100. doi:10.1177/1087054707305152.

Martin, G., Copeland, J., Gilmour, S., Gates, P. y Swift, W. (2006). The Adolescent Cannabis Problems Questionnaire (CPQ-A): psychometric properties. Addictive Behaviors, 31, 2238-2248. doi:10.1016/j.addbeh.2006.03.001.

Martin, W.R., Sloan, J.W., Sapira, J.D. y Jasinski, D.R. (1971). Physiologic, subjective, and behavioral effects of amphetamine, methamphetamine, ephedrine, phenmetrazine, and methylphenidate in man. Clinical Pharmacology and Therapeutics, 12, 245-258. doi:10.1002/cpt1971122part1245.

Mezquita, L., Stewart, S.H., Ibáñez, M.I., Ruipérez, M.A., Villa, H., Moya, J. y Ortet, G. (2011). Drinking motives in clinical and general populations. European Addiction Research, 17, 250-261. doi:10.1159/000328510.

Miller, W.R. y Rollnick, S. (1991). Motivational interviewing: Preparing people to change addictive behavior. New York: Guilford Press.

Millon, T. (1993). Manual of Millon Adolescent Clinical Inventory. Systems NC, editor. Minneapolis.

Millon, T. (2004). MACI: inventario clinico para adolescentes de millón. Ediciones TEA, editor. Madrid.

Monk, R.L. y Heim, D. (2016). Expectativas relacionadas con el alcohol en adultos y adolescentes: semejanzas y diferencias. Adicciones, 28, 35-40. doi:10.20882/adicciones.788.

Muñiz, J., Elosua, P. y Hambleton, R.K. (2013). Directrices para la traducción y adaptación de los tests: segunda edición. Psicothema, 25, 151-157. doi:10.7334/psicothema2013.24. 
Observatorio Español sobre Drogas. Encuesta estatal sobre el consumo de drogas en enseñanzas secundarias (ESTUDES) (2014). Observatorio Español de las Drogas y las Adicciones, Madrid.

Scherrer, J.F., Grant, J.D., Duncan, A.E., Sartor, C.E., Haber, J.R., Jacob, T. y Bucholz, K. K. (2009). Subjective effects to cannabis are associated with use, abuse and dependence after adjusting for genetic and environmental influences. Drug and Alcohol Dependence, 105, 76-82. doi:10.1016/j.drugalcdep.2009.06.014.

Simons, J., Correia, C.J., Carey, K.B. y Borsari, B.E. (1998). Validating a five-factor marijuana motives measure: Relations with use, problems, and alcohol motives. Journal of Counseling Psychology, 45, 265-273. doi:10.1037/00220167.45.3.265.

Simons, J., Correia, C.J. y Carey, K.B. (2000). A comparison of motives for marijuana and alcohol use among experienced users. Addictive Behaviors, 25, 153-160. D: doi:10.1016/S0306-4603(98)00104-X.

Simons, J.S., Gaher, R.M., Correia, C.J., Hansen, C.L. y Christopher, M.S. (2005). An affective-motivational model of marijuana and alcohol problems among college students. Psychology of Addictive Behaviors, 19, 326-334. doi:10.1037/0893-164X.19.3.326.

Tragesser, S.L., Trull, T.J., Sher, K.J. y Park, A. (2008). Drinking motives as mediators in the relation between personality disorder symptoms and alcohol use disorder. Journal of Personality Disorders, 22, 525-537. doi:10.1521/ pedi.2008.22.5.525.

Thornton, L.K., Baker, A.L., Lewin, T.J., Kay-Lambkin, F.J., Kavanagh, D., Richmond, R.,... Johnson, M.P. (2012). Reasons for substance use among people with mental disorders. Addictive Behaviors, 37, 427-434. doi:10.1016/j. addbeh.2011.11.039.

Zvolensky, M.J., Vujanovic, A., Bernstein, A., Bonn-Miller, M.O., Marshall, E.C. y Leyro, T.M. (2007). Marijuana use motives: A confirmatory test and evaluation among young adult marijuana users. Addictive Behaviors, 32, 3122-3330. doi:10.1016/j.addbeh.2007.06.010.

Zeiger, J.S., Haberstick, B.C., Corley, R.P., Ehringer, M.A., Crowley, T.J., Hewitt, J. K. y Rhee, S. (2012) Subjective effects to marijuana associated with marijuana use in community and clinical subjects. Drug and Alcohol Dependence, 123, S52-S58. doi:10.1016/j.drugalcdep.2012.02.014.

Zumbo, B.D., Gadermann, A.M. y Zeisser, C. (2007). Ordinal versions of coefficients alpha and theta for Likert rating scales. Journal of Modern Applied Statistical Methods, 6, 21-29. doi:10.1016/j.drugalcdep.2009.12.026. 